\section{Achtung! Neu für Spitalbetreiber und Klinikbesitzer}

K. F. Bösch

\begin{abstract}
Wenn der Patient, heute Kunde oder Leistungsempfänger genannt, zufrieden ist, läuft das Geschäft. Betrachten Sie das bitte ja nicht als Gemeinplatz. Das KVG schreibt messbare, nachweisbare Qualität vor. Patientenzufriedenheit gehört offenbar zu diesen Messgrössen. Mit zufriedenen Kunden gewinnen Sie nicht nur neue dazu, sondern die Aussicht steigt, als qualitätsbetonter und kundenorientierter Betrieb akkreditiert zu werden. Nebenbei gilt es auch, wichtige Wettbewerbsfaktoren und Marketingaspekte zu berücksichtigen, damit der Kunde nicht zum nächsten Anbieter überläuft, der ihn abwirbt. Die Abwerbung kann diesem - leider - mit entsprechenden besseren Aushängeschildern auch dann gelingen, wenn er eigentlich weniger gut ist. Das gehört zu den Gesetzen des Marktes.

Und die Forschung (so zumindest nennt man dieses Tun in gewissen Kreisen) hat zutage gebracht, um was es hier, im Spitalbereich, geht. Zufrieden ist er, der Kunde, nämlich, wer hätte das gedacht, wenn er geheilt wurde oder wenn es ihm bei der Entlassung wenigstens besser geht als beim Eintritt, wenn er nicht zu lange hospitalisiert war, wenn das Essen gut, das Zimmer hell und sauber, die Pflegenden aufopfernd, fröhlich und lieb und die Ärzte mitteilsam, kompetent und einfühlsam waren. So ist es, man
\end{abstract}

Korrespondenz:

Dr. med. Kurt F. Bösch

Schlossbach

CH-9404 Rorschacherberg

E-mail: kuboesch@paus.ch glaubt es kaum. Wer sagt da klar, selbstverständlich, binsenwahr? Solches wurde und wird laufend erforscht, sonst wäre ja niemand darauf gekommen. Natürlich fühlt sich der Kundenpatient, das sei zusätzlich erwähnt, weil auch erforscht, nicht selten dann zufrieden(er), wenn es ihm wenigstens besser geht als dem Bettnachbarn. Wenn obiges ganz oder teilweise nicht zutrifft, kann es leicht vorkommen, dass der Patientenkunde unzufrieden ist. Unzufrieden mag er auch sein, wenn Geschlecht, Alter und Aussehen des Arztes oder des Pflegepersonals nicht seinen Vorstellungen entsprechen. Dagegen lässt sich schwer etwas unternehmen. Unzufrieden kann er auch sein, wenn er als sozial besser Gestellter eventuell höhere Anforderungen stellt. Solche Imponderabilien sind zwar begreiflich, erklärbar, aber es ist schwierig, ihnen zu begegnen.

Die vorliegenden Arbeiten, die mir zu denken geben, sprechen übrigens teils von der Zufriedenheit mit dem Arzt und teils von der Zufriedenheit mit dem Spitalbetrieb. Man nimmt es da nicht so exakt. Und doch ist es nicht ganz dasselbe.

Ganz besonders weise ist die Feststellung: Die Zufriedenheit muss - so können die lernen, welche das nicht schon gemerkt haben - nicht etwa kongruent mit einer guten Betreuung und Behandlung sein. Die Zufriedenheit folgt nämlich anderen Gesetzen. Was bleibt uns da noch vorzukehren?

Und überhaupt, lernen wir weiter: Niemand weiss, ob der, welcher glaubt zufrieden $\mathrm{zu}$ sein, auch tatsächlich zufrieden ist. Was es zu berücksichtigen gilt. Denn es steht zu lesen: «Des Patienten eigene Beurteilung (subjektiv und objektiv) aller Aspekte einer medizinischen Versorgung beinhaltet auch die zwischenmenschlichen Gesichtspunkte einer Behandlung und deren organisatorischen Ablauf."

Ich glaube rufen $\mathrm{zu}$ hören. Ich habe mich getäuscht, niemand rief. Das war vor vielen Jahren, als Angelus Silesius (ca. 1624-1677) rief: «Mensch, werde wesentlich!» Es gab damals noch kein totales Qualitätsmanagement (TQM).

PS: Ich verkneife es mir, die Arbeiten zu zitieren, auf die ich verweise. Sollte in der vorstehenden Glosse eine Meinung durchschimmern, ist die Übereinstimmung (oder ihr Gegenteil) mit der Meinung anderer Personen oder Gruppierungen rein zufällig. 